\title{
„Medaillen sind für mich ein kleines Denkmal“
}

\author{
Marianne Dietz im Gespräch mit Kristina Vaillant
}

\begin{abstract}
Am 14. September 2020 wurde der Mathematiker Moritz Kerz während der Eröffnungszeremonie der Jahrestagung der DMV an der TU Chemnitz mit der Minkowski-Medaille ausgezeichnet. Weil die bronzene Medaille zum ersten Mal verliehen wurde, erzählt ihre Gestalterin, die Berliner Bildhauerin Marianne Dietz, von der Entstehung des Kunstwerks und von einer ganz eigenen künstlerischen Disziplin.
\end{abstract}

Frau Dietz, ich nehme an, als Bildhauerin gestalten sie hauptsächlich figürliche Plastiken, wie sind Sie zu den Medaillen gekommen?

Es stimmt, die figürliche Bildhauerei ist der Ursprung. Aber die Medaillen spielen bei mir eine zunehmend große Rolle. In den letzten Jahren habe ich eigentlich hauptsächlich Medaillen gemacht, mit Vorliebe Porträtmedaillen wie die Minkowksi-Medaille. Sie sind im Grunde genommen ein „Denkmal im Kleinformat“, eine Würdigung von Menschen in unserer Gesellschaft, die etwas Bemerkenswertes geleistet haben und die es verdienen, dass man ihnen eine bleibende Erinnerung schafft.

Sind das immer Auftragsarbeiten, so wie die Minkowski-Medaille?

Nein, aber es wäre schön, wenn es solche Auftragsarbeiten häufiger geben würde. Ein ganz großer Teil sind freie Arbeiten, die ich mir selber suche. Zum Beispiel habe ich eine Medaille für Maryam Mirzakhani gestaltet, die als erste Frau die Fields-Medaille bekommen hat.

Wie kam es dazu?
Der Anlass war eine Ausschreibung der Fédération Internationale de la Médaille d'Art (FIDEM), die alle zwei Jahre stattfindet. 2017 war „Frauen in der Wissenschaft" das Wettbewerbsthema. Da habe ich Maryam Mirzakhani entdeckt. Es gibt Videos von ihren Vorlesungen, die haben mich beeindruckt. So eine junge, intelligente, charismatische Frau, die mit so viel Begeisterung unterrichtet hat, das finde ich einfach klasse. Ich war bestürzt, von ihrem frühen Tod zu lesen, nachdem ich die Medaille fertiggestellt hatte.

Der Mathematiker Hermann Minkowski ist schon Anfang des 20. Jahrhunderts gestorben. Wie sind Sie vorgegangen, als Sie ihn porträtiert haben?

Mit dem Auftrag wurde mir ein Konvolut von Bildern zugetragen. Aber letztlich gibt es nicht so viele unterschiedliche, denn zu seiner Zeit wurde noch wenig fotografiert. Die Auswahl hat sich sehr schnell eingeschränkt und ich habe das Bild ausgesucht, das sich am besten für die Medaille geeignet hat.

Welchen Kriterien muss es genügen?

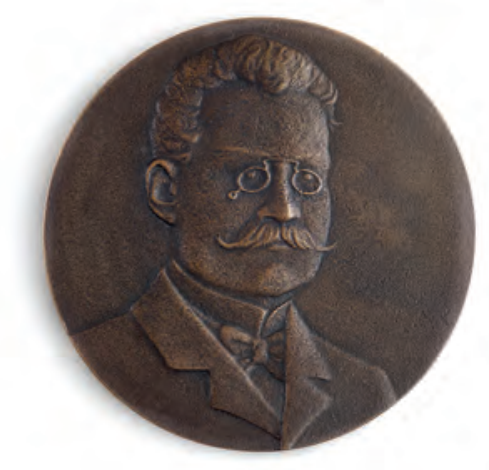


Am Wichtigsten ist die Wiedererkennbarkeit. Die klassische Porträtmedaille zeigt eigentlich das absolute Profil, einen Schattenriss. Das ist die individuelle Initiale der Person, die wie bei antiken Münzen auch nach starker Abnutzung lesbar bleibt. So ein Bild gibt es von Minkowski nicht. Das war aber kein Problem, denn die gewählte Fotografie eignet sich sehr gut für ein räumlich plastisches Portrait.

Anfang März dieses Jahres haben Sie den Auftrag bekommen, im Mai wurde die Medaille gegossen. Wie haben sie auf Grundlage der Fotovorlage diese kleine Reliefplastik geschaffen?

Ich bin vorgegangen wie immer. Nach dem ausgewählten Foto mache ich zuerst eine Zeichnung mit Bleistift. Dann setze ich am Rechner die Zeichnung in ein Layout und gestalte verschiedene Entwürfe, so dass ich den Auftraggebern demonstrieren kann, wie unterschiedlich es aussieht, wenn man beispielsweise den Text mit auf der Vorderseite oder alternativ auf der Rückseite anordnet. Wenn der Entwurf feststeht, wird die Zeichnung mit Kohlepapier auf eine Gipsscheibe übertragen. Auf diese Gipsscheibe lege ich dann eine leicht transparente Wachsscheibe und schneide sie entlang der Kontur der Zeichnung aus. So bekomme ich das erste Volumen, mit dem ich plastisch arbeiten kann. Das heißt, es wird geschabt und modelliert. Als nächstes wird das Wachsmodell in Gips abgegossen. Nun hat man ein Negativ, an dem durch Aushöhlen fehlende Masse an das Relief angetragen werden kann. Aus diesem Negativ wird wieder ein Positiv gegossen, das weiter bearbeitet und wieder abgegossen wird. Es ist ein rein subtraktives Arbeiten. Wenn ich im Gipsnegativ arbeite, trage ich Volumen an und wenn ich im Positiv arbeite, trage ich Volumen ab. In der Folge gießt man so oft um, bis der Zustand erreicht ist, der zufrieden stellt. Insgesamt habe ich für die Portraitseite der Minkowski-Medaille bis zum fertigen Modell zehn Abgüsse gemacht.

Welche Werkzeuge benutzen Sie beim Modellieren?

Ich nehme das, was ich finde. Das können Nadeln, Messer oder kleine Fräserköpfe sein, die ich in einen Mienenstift einsetze.

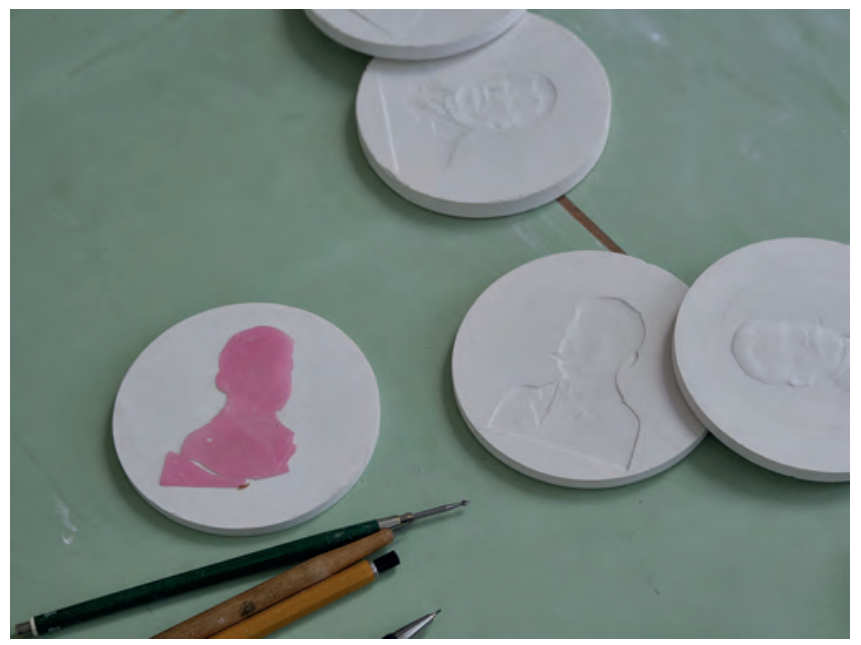

Und wenn Ihre Arbeit getan ist?

Dann gießt eine Traditionsgießerei in Schöneiche bei Berlin die Medaillen in Bronze und zwar im Sandgussverfahren. Das Gipsmodell der Minkowski-Medaille liegt jetzt beim Gießer und bleibt dort auch. Von diesem Modell wird jede Folgemedaille direkt abgeformt. So ist gewährleistet, dass jede der Minkowski-Medaillen gleich aussieht. Wenn die nächste Medaille gebraucht wird, muss ich nur rechtzeitig Bescheid wissen, dann gebe ich dem Gießer den Auftrag.

\section{Und wer macht die Gravur?}

Die Gravur kann ich nicht selbst machen. Ich habe einen Kollegen, Bodo Broschat, der noch an der staatlichen Münze in der DDR gelernt hat. Er hat den Namen des Preisträgers nach meiner Vorlage auf der Rückseite graviert, und es ist wirklich hervorragend gelungen.

Dass Medaillen eine "Sonderform der Reliefplastik" und auch heute noch eine eigene Kunstform sind, war mir vor unserem Gespräch nicht klar. Welche Rolle spielt diese „Kleinkunst“ im Kunstbetrieb?

In der öffentlichen Wahrnehmung sind wir Medailleure eigentlich gar nicht vorhanden. Ein Problem ist, dass diese Kunstform, ebenso wie die klassische Bildhauerei, an den Kunsthochschulen keine Rolle mehr spielt und nicht mehr gelehrt wird. So wird leider kaum noch Nachwuchs ausgebildet.

\section{Woran liegt das?}

Ich vermute, dass der Kunstbetrieb, der auf große Effekte und plakative Objekte aus ist, den Markt so beherrscht, dass der Mensch kein Auge mehr für die stillen, kleinen Dinge hat. Für mich persönlich hat das Medaillenarbeiten etwas sehr Meditatives. Wenn ich in diese kleine Welt eintauche, dann verschwindet alles um mich herum, ich versinke in der Form. So ähnlich ist es, wenn man das Berliner Münzkabinett im Bode-Museum auf der Museumsinsel besucht, seine Augen auf die kleinen Teile richtet und entdeckt, was für eine große Welt sich da öffnet. Wie viel Mühe, Geduld und Begabung Menschen schon immer aufgebracht haben, Kleinode mit Bedeutung zu schaffen.

Wird während der Arbeit an der Medaille auch der Mensch, den Sie damit würdigen, noch einmal lebendig?

Ja, das ist das Schöne. Man beschäftigt sich dann mit der Person, soweit es möglich ist. Sie tritt quasi in das Leben wie eine neue Bekanntschaft.

Was für eine Person ist der Mathematiker Minkowski für Sie?

Wie soll ich sagen, sein Metier ist mir fremd und seine mathematischen Ideen und Leistungen sind für mich unbegreiflich. Insofern bleibt er mir verschlossen. Er war schon in jungen Jahren ein beeindruckend kluger Kopf mit außergewöhnlichem Forscherdrang. Absolut respektabel. 

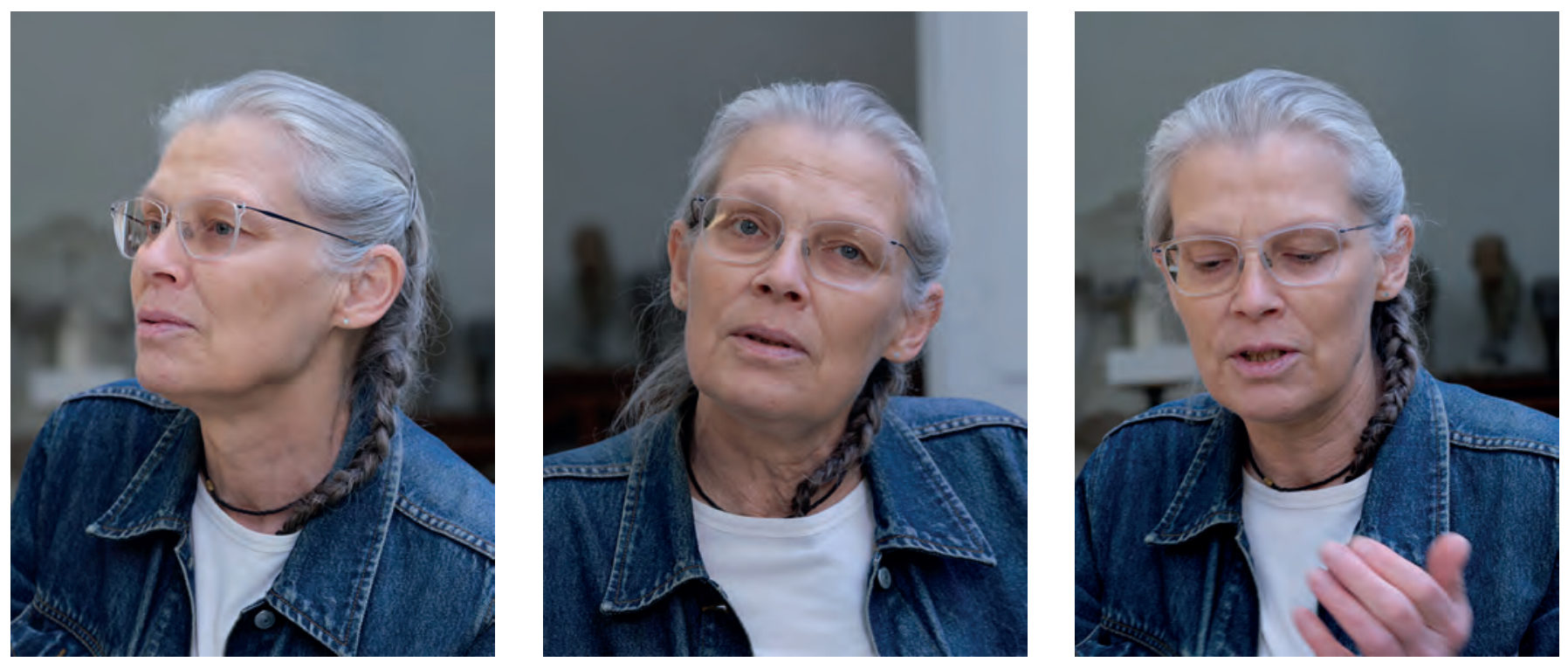

Haben Sie als Künstlerin eine besondere Beziehung zur Mathematik?

Also die Mathematik selbst sprengt schnell mein Vorstellungsvermögen. Ich arbeite intuitiv. Aber so ein Regelwerk, so eine Systematik, die ja auch mit Harmonien zu tun hat, finde ich grundsätzlich faszinierend.

Die Porträtmedaille von Maryam Mirzakhani ist mittlerweile Teil der Sammlung des Münzkabinetts der Staatlichen Museen in Berlin. Wird man dort bald auch eine Kopie der Minkowski-Medaille bewundern können?
Ich werde sie auf jeden Fall anbieten und es wäre sehr schön, wenn sie ebenfalls in der Sammlung Platz findet.

Wo der diesjährige Preisträger Moritz Kerz seine Minkowksi-Medaille aufbewahrt, wissen wir nicht. Was würden Sie ihm empfehlen?

Wenn sie ihm gefällt, dann damit leben, auf den Schreibtisch legen oder aufs Fensterbrett, da gibt es manchmal gutes Streiflicht. Auf jeden Fall sollte er sie ab und zu ruhig in die Hand nehmen.

Frau Dietz, ich danke Ihnen für dieses Gespräch. 